\title{
Silicon Photomultipliers as a Low-Cost Fluorescence Detector for Capillary Electrophoresis
}

\author{
Brae V. Petersen ${ }^{1,2}$, Luke Gallion ${ }^{1,2}$, Nancy L. Allbritton ${ }^{1 *}$. \\ 1. Department of Bioengineering, University of Washington, Seattle, Washington 98195, United States \\ 2. Department of Chemistry, University of North Carolina at Chapel Hill, Chapel Hill, North Carolina 27599, United States
}

ABSTRACT: Capillary electrophoresis (CE) is a highly efficient separation method capable of handling small sample volumes $(\sim \mathrm{pL})$ and low ( yoctomole) detection limits, and as such is ideal for applications that require high sensitivity such as single-cell analysis. ${ }^{1-3}$ Low-cost CE instrumentation is quickly expanding but low-cost, open-source fluorescence detectors with ultra-sensitive detection limits are lacking. ${ }^{4-6}$ Silicon photomultipliers (SiPM) are inexpensive, low-footprint detectors with the potential to fill the role as a detector when cost, size, and customization are important. In this work we demonstrate the use of a SiPM in CE with zeptomolar detection limits and a dynamic range spanning five orders of magnitude, comparable to photomultiplier detectors. The performance of these detectors was measured using a constant wave excitation laser in an epifluorescence detection configuration. We characterize the performance of the SiPM as a highly sensitive detector by measuring enzyme activity in single cells. This simple, small footprint, and low-cost $(<\$ 130)$ light detection circuit will be beneficial for open-source, portable, and budget friendly instrumentation requiring high sensitivity.

Cell heterogeneity has become increasingly recognized as an important factor in many aspects of pathology, immunology, and stem-cell biology. ${ }^{7,8}$ As a result, a myriad of analytical tools have been developed to evaluate this heterogeneity. ${ }^{2,3}$ Capillary electrophoresis (CE) with fluorescence detection is one such tool that has been widely utilized in the academic laboratory to analyze single cells, largely due to the high sensitivity and large dynamic range of fluorescent detectors. ${ }^{2,3}$ Zeptomole $\left(10^{-21}\right)$ detection limits are typical with some setups capable of achieving yoctomole $\left(10^{-24}\right)$ limits of detection (LOD). ${ }^{9}$ Furthermore, these instruments are well suited for chemical cytometry since CE instruments can handle small $(\sim \mathrm{pL})$ sample volumes, and therefore single-cell lysates do not experience significant dilution prior to assay. However, CEbased chemical cytometry systems have not seen widespread use clinically, partially due to the high cost, large instrument footprint, and high technical expertise requirements. As such, instrument modifications to address these issues should be explored.

Several fluorescent detection setups have been described for chemical cytometry, many of which have employed photomultiplier tubes (PMTs). ${ }^{9-11}$ PMTs work using a photocathode housed within a vacuum environment to transduce light into an electrical signal. PMTs have high gain $\left(10^{5-9}\right)$ and maintain linearity over a large dynamic range, typically as many as 6 orders of magnitude. ${ }^{10,12}$ For these reasons PMTs are commonly utilized in CE systems where cost is not a driving factor. With the development of low-cost open-source CE setups, there is a desire for an inexpensive, open-source detector that can be customized according to the need of the instrument or project. ${ }^{6}$

Silicon photomultipliers (SiPM) are significantly less expensive than PMTs but have similar gains $\left(10^{5-6}\right)$ and have been suggested for use as fluorescence detectors for low-cost
CE instruments. ${ }^{5,12}$ However, applications using SiPMs as a fluorescence detector in analytical instruments have only recently been explored. ${ }^{13-15}$ SiPMs consist of arrays of micron sized photodiodes (i.e., 50x50 $\mu \mathrm{m}$ dimensions) that are biased as single-photon avalanche diodes (SPAD). ${ }^{16}$ Each microcell produces a digital current signal when struck by a photon, with the entire array producing an analog signal at higher light densities. ${ }^{12}$ Several methods have emerged for deploying a SiPM in experiments for photon counting where a pulsed light source and high bandwidth analog to digital converter (ADC) or oscilloscope is used. ${ }^{17,18}$ Low frequency or constant power applications have also begun to emerge for projects focused on providing affordable instrumentation. ${ }^{13,19,20}$ We sought to apply a simple circuit for the SiPM detector for use with a detection apparatus and signal processing workflow designed for oncolumn epifluorescence detection. By doing so, we found the SiPM can obtain a molar LOD of $10^{-21}$ moles and span a linear dynamic range of 5-6 orders of magnitude, thus justifying. SiPM use in affordable chemical cytometry instrumentation and particularly CE-based instruments. The detection circuitry is both low-cost $(\sim 130)$ and relatively simple, making it ideal for a fluorescence detector for affordable and open-source instrumentation.

\section{EXPERIMENTAL}

\section{Materials and Reagents}

Sphingosine fluorescein (SF) and sphingosine 1-phosphate fluorescein (S1PF) were purchased from Echelon Biosciences Inc. (Cat. \# S-100F and \#S-200F, Salt Lake City, UT). Trizma-base, 4-(2-hydroxyethyl)-1-piperazineethanesulfonic acid (HEPES), 1-propanol, potassium chloride, and magnesium chloride were purchased from Millipore Sigma. Tween 20 was purchased from BioRad Laboratories. Glass slides, calcium 

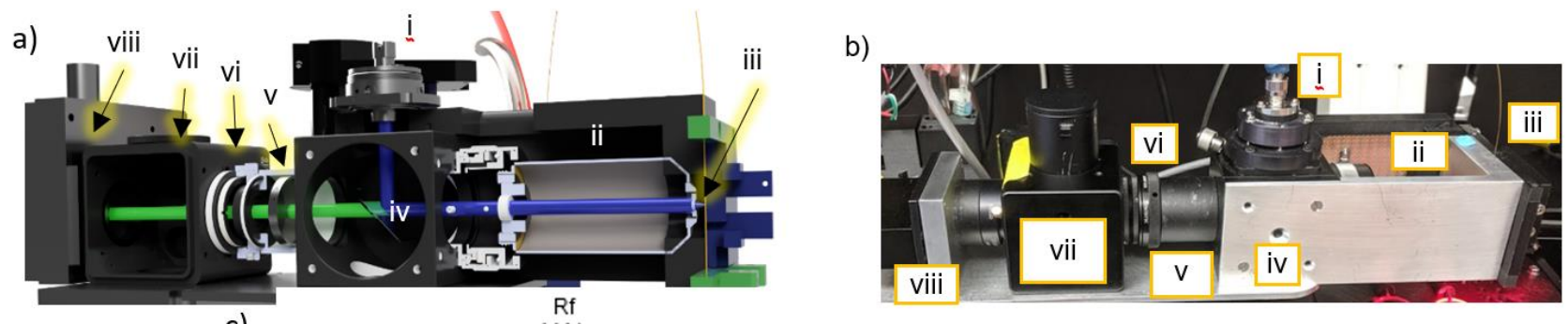

c)

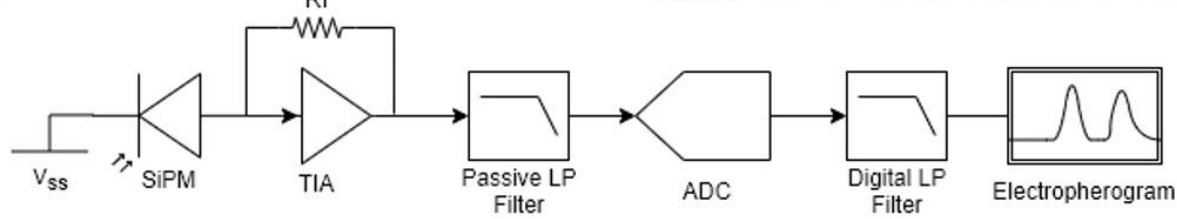

Figure 1. CE fluorescence detection assembly and simplified pathway. Diagram (a) and photo (b) of the optical pathway used for testing the detectors. Excitation light from a $488 \mathrm{~nm} \mathrm{CW}$ laser is collimated by a fiber optic coupler (i). A dichroic beam splitter (iv) reflects the light to a 0.75 NA 40x objective (ii) which then focuses the light onto the capillary (iii). Emission light (from fluorophores within the capillary lumen) travel through the beam splitter (iv) and this emitted light is filtered using a $520 \mathrm{~nm}$ low pass filter (v). Stray light is spatially filtered using an iris (vi). A 4-way optic mount (vii) is used to mount the detectors (viii) to the optical path. c) Signal flow chart from SiPM to final electropherogram. A negative current is generated by the SiPM as light strikes the microcells. An inverting transimpedance amplifier converts current into a voltage using the feedback resistor, Rf. The amplifier output is filtered by a passive low-pass filter then oversampled at $75 \mathrm{kHz}$ using an ADC. The oversampled signal is averaged to 8 $\mathrm{Hz}$ and a Butterworth digital filter applied to the $8 \mathrm{~Hz}$ data to obtain the final electropherogram (time vs relative fluorescence)

chloride, sodium phosphate was purchased from Fisher Scientific. Dulbecco's modified eagle's medium (DMEM) and fetal bovine serum (FBS) were from ThermoScientific (Waltham, MA).

\section{Instrumentation}

For this work, a modular CE detection assembly using epiillumination was designed and built (Figure 1). The detection window is created by removing the capillary coating $4 \mathrm{~cm}$ from the capillary inlet using a flame. The detection window is aligned perpendicular to the light path within the detection objective using 3D printed levers secured with $2 \mathrm{M}$ threaded screws to adjust the capillary position. Excitation light from an optical fiber pigtail coupled to a $488 \mathrm{~nm}$ constant wave (CW) laser is collimated using a fiber receptacle collimator $(\mathrm{OZ}$ Optics, Ottawa, Canada) and directed to the detection objective using a $488 \mathrm{~nm}$ dichroic filter. A 0.75 numeric aperture (NA) 40x objective focuses incoming excitation light to the capillary lumen. The emission light is collimated by the objective and passes through a dichroic, a $525 \mathrm{~nm}$ bandpass filter, and $6.5 \mathrm{~mm}$ iris before striking the detector. The detectors are attached to a four-way optical block and can be exchanged without changing the alignment of the capillary or other aspects of the CE detection assembly. A $3.7 \times 13 \mathrm{~mm}$ side on PMT (H9306, Hamamatsu, Bridgewater, NJ) was used to provide a comparison to the SiPM detectors. A parts list for the detection assembly is available in the supplemental information (Table S1).

The custom CE instrument used for this work incorporates characteristics of traditional $\mathrm{CE}$ as well as modifications to improve capillary positioning and control for single-cell analysis. ${ }^{11}$ An overview of the custom instrument is provided in supplemental (Figure S1).

\section{SiPM Detector}

SiPM detectors (MICROFC -30035, -30050, and -60035) were procured from OnSemiconductor (Phoenix, AZ). Printed circuit boards (PCB) were designed using an opensource electronics design software suite (KiCad EDA 5.1. http://kicadpcb.org/, March 6, 2020) and fabricated by BasicPCB (Aurora, Colorado, USA). The SiPM circuit (Figure S2) and parts list (Table S2) are provided in the supplemental along with Gerber files for the PCB fabrication and a complete bill of materials. The manufacturer's recommendations were followed for assembly. The SiPM chip was negatively biased 1-5 V above the breakdown voltage. Measurements were taken at $1 \mathrm{~V}$ intervals. The signal from the detectors were filtered using a passive low-pass hardware filter $\left(5^{\text {th }}\right.$ order elliptical, $1 \mathrm{~dB}$ at $1 \mathrm{kHz}$, see Table S1). Oversampling filtering was performed at $75 \mathrm{kHz}$ and averaged to achieve a final sampling rate of $8 \mathrm{~Hz}$ to reduce random noise. A $2^{\text {nd }}$ order digital butter filter with a cutoff of $1.5 \mathrm{~Hz}$ was applied twice (forwards and backwards) to the $8 \mathrm{~Hz}$ electropherogram.

\section{Limits of Detection}

The signal to noise ratio (SNR) was measured for each of the detectors. The signal was measured from a $41 \mathrm{nM}$ fluorescein solution loaded into the capillary by gravity $(3 \mathrm{~cm}$ height difference for $3 \mathrm{~s})$. The total injection volume $(1.0 \mathrm{~nL})$ was calculated using the volume loaded due to gravity plus the volume loaded due to diffusion and spontaneous fluid displacement. ${ }^{21}$ To measure spontaneous fluid displacement, the average corrected area from $3 \mathrm{~cm}$ gravity injections $(n=7)$ was compared to the average corrected area from $0 \mathrm{~cm}$ gravity injections $(n=7)$. The volume due to spontaneous fluid loading was calculated according to Equation 1, where CA is the corrected area for the measured $0 \mathrm{~cm}$ and $3 \mathrm{~cm}$ injections, and $\mathrm{V}_{3 \mathrm{~cm}}$ is the volume loaded into the capillary by gravity:

$$
\text { Equation 1: } V_{s f d}=\frac{C A_{0 \mathrm{~cm}}}{C A_{3 \mathrm{~cm}}-C A_{0 \mathrm{~cm}}} V_{3 \mathrm{~cm}}
$$




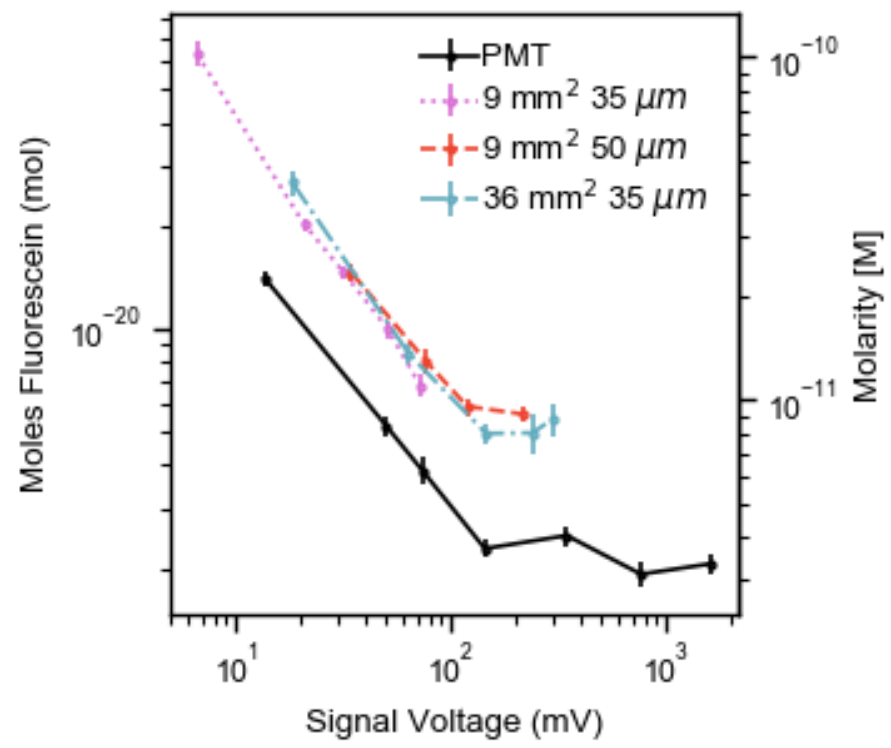

Figure 2. Estimated limit of detection (LOD) from the SNR for fluorescein as a function of signal voltage $(\mathrm{mV})$ for each detector. LOD was estimated as the minimal signal detected at $3 \mathrm{x}$ noise. On the $\mathrm{x}$-axis, the signal voltage corresponds to the maximum voltage for the fluorescein peak after subtracting the noise. The moles detected is estimated from the total volume loaded into the capillary $(1.0 \mathrm{~nL})$.

At this volume, the total amount of fluorescein loaded into the capillary was $2.5 \times 10^{-17}$ moles. The noise was calculated using the standard deviation across 40 consecutive points (corresponding to a time window of $5 \mathrm{~s}$ ) of the electropherogram with the least detectable signal. A signal of $3 x$ the noise was used to calculate the molar LOD for each sensor.

\section{Dynamic Range}

Fluorescein standards were prepared to create a calibration curve near the saturation point for the detectors. Fluorescein standards were prepared using a 1:3 serial dilution in tris buffer $(100 \mathrm{mM}, \mathrm{pH} 8.1$, conductivity $5.3 \mathrm{mS} / \mathrm{cm})$ from $10 \mu \mathrm{M}$ stock solution. The peak areas were integrated from the filtered electropherograms using a trapezoid approximation function. Corrected areas were calculated by dividing the peak area by the corresponding retention time.

\section{CE Separations}

A $50 \mu \mathrm{m}$ inner diameter, $375 \mu \mathrm{m}$ outer diameter capillary (\#1068150017, Molex, Lisle, IL) with a total length of $45 \mathrm{~cm}$ and a detection length of $4 \mathrm{~cm}$ was used. Buffer conductivity was measured using a conductivity meter (\#4360, Traceable, Webster, TX). At the start of the day, the capillary was conditioned by rinsing for $10 \mathrm{~min}$ with $0.1 \mathrm{M} \mathrm{HCl}, 30 \mathrm{~min}$ with $1 \mathrm{M}$ $\mathrm{NaOH}$, followed by 5 min rinses with $\mathrm{H}_{2} \mathrm{O}$, and separation buffer. Prior to each separation the capillary was rinsed with separation buffer for $30 \mathrm{~s}$. All separations were performed with an electric field strength of $333 \mathrm{~V} / \mathrm{cm}$ across the capillary. For the fluorescein separations the separation buffer consisted of $100 \mathrm{mM}$ tris buffered to $\mathrm{pH} 8.0$ with a buffer conductivity of $5.3 \mathrm{mS} / \mathrm{cm}$.

For the sphingolipid separations $30 \mu \mathrm{m}$ inner diameter, 375 $\mu \mathrm{m}$ outer diameter capillary (\#1068150013, Molex, Lisle, IL) with a total length of $60 \mathrm{~cm}$ and a detection window at $5 \mathrm{~cm}$ was used. the separation buffer consisted of $27 \mathrm{mM}$ phosphate at $\mathrm{pH} 7.4$ and $10 \%$ n-propanol with a conductivity of 3.0

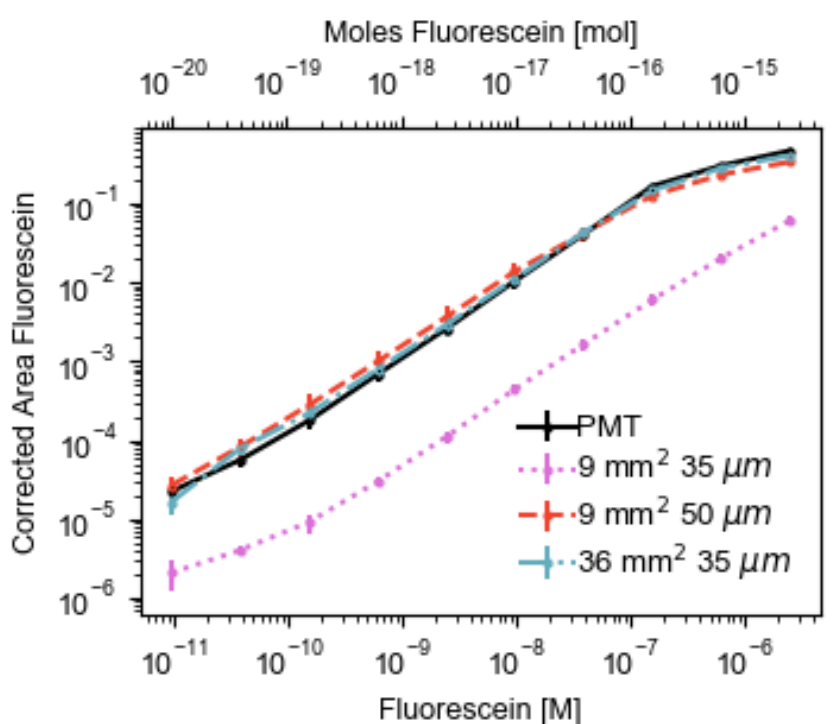

Figure 3. Comparison of the dynamic range for the SiPM and PMT detectors. A fluorescein calibration curve with concentrations from $2.5 \mu \mathrm{M}$ to $9 \mathrm{pM}(\mathrm{n}=3)$ was measured with each detector. SiPM bias voltages were set to their maximum values $(29.5$ V) while the PMT was set to $300 \mathrm{mV}$ corresponding to its maximum SNR before plateauing (Figure 2).

$\mathrm{mS} / \mathrm{cm}$. An electric field strength of $250 \mathrm{~V} / \mathrm{cm}$ was applied during electrophoresis. SF and S1PF were identified by comparison of their migration times to standards while hexadecenoic acid fluorescein $\left(\mathrm{HAF}^{*}\right)$ was identified from its elution order based prior assays with SF.

\section{Cell Preparation}

The K-562 cell line was obtained from the American Type Culture Collection (ATCC) (CRL-1469, ATCC, Manassas, Virginia) and maintained in a humidified atmosphere of $37^{\circ} \mathrm{C}$ in $5 \% \mathrm{CO}_{2}$. Cells were cultured in Dulbecco's Modified Eagle Medium (DMEM) supplemented with $10 \%$ fetal bovine serum (FBS), penicillin (100 units/mL), and streptomycin (100 $\mu \mathrm{g} / \mathrm{mL})$.

SF was loaded into cells by incubating $5 \times 10^{5}$ cells in 100 $\mu \mathrm{L}$ culture media containing freshly diluted $10 \mu \mathrm{M}$ SF for 30 min as described previously. ${ }^{21}$ Cells were stored at $37^{\circ} \mathrm{C}$ in a $5 \%$ carbon dioxide atmosphere during incubation with SF. Cells were centrifuged (700 x g, $2.5 \mathrm{~min}$ ) and then washed $2 \mathrm{x}$ with $200 \mu \mathrm{L}$ physiologic cell buffer ( $135 \mathrm{mM}$ sodium chloride, $5 \mathrm{mM}$ potassium chloride, $1 \mathrm{mM}$ magnesium chloride, $1 \mathrm{mM}$ calcium chloride, and $10 \mathrm{mM}$ HEPES at $\mathrm{pH}$ 7.4). An aliquot of 10,000 cells was removed and overlaid onto a glass slide with $600 \mu \mathrm{L}$ physiologic buffer.

\section{RESULTS AND DISCUSSION}

\section{Limits of Detection}

SiPM detectors are arrays of micron-sized photodiodes, referred to as microcells, connected in parallel. These microcells are operated in Geiger mode above their breakdown voltage. As a photon strikes a photodiode, a current pulse is produced. At high light levels, hundreds or thousands of photodiodes are simultaneously activated, and their total current can be recorded as a DC analog signal using low-cost electrical components. ${ }^{12,16}$ While SiPMs can be used in photon counting mode, the excitation light that reached the detector for this setup was 
too high to employ photon counting. For this reason, only low bandwidth DC measurements from the SiPMs were measured. Of the factors that may influence performance, we hypothesized fill factor would considerably influence SiPM signal strength and sensitivity, as a greater fill factor correlates with a greater probability a photon will strike a photo-responsive area. ${ }^{12}$ Fill factor increases as the microcell size increases since fewer microcells are needed for the same area and thus require fewer circuit structures (fabricated wires and resistors) for the array. Sensors with different SPAD microcell sizes as well as total array sizes were tested to gauge which SiPM configuration leads to the greatest SNR.

The LOD of the SiPMs and PMT were compared using a $\mathrm{CE}$ detection pathway configured in an epifluorescence format (Figure 1). A feature of this design and housing is that the detector can be easily removed and then re-inserted without modifying the capillary alignment facilitating direct comparison between the different fluorescence detectors. In addition to the three SiPMs, a PMT previously measured to possess zeptomole detection limits was used as a reference detector. ${ }^{21}$ LODs of three SiPM sensors were measured: a $9 \mathrm{~mm}^{2}$ sensor area with $50 \mu \mathrm{m}$ microcells, a $9 \mathrm{~mm}^{2}$ sensor area with $35 \mu \mathrm{m}$ microcells, and a $36 \mathrm{~mm}^{2}$ sensor area with $35 \mu \mathrm{m}$ microcells. The SNR of each sensor (Figure S3) was used to determine the $3 \sigma$ limit of detection as a function of the peak height (Figure 2). A sensor with a lower peak height at an equivalent SNR will be able to utilize more of the op-amp and ADC dynamic range. Of the SiPM detectors the $36 \mathrm{~mm}^{2}$ sensor was the most sensitive, followed by the 50 and $35 \mu \mathrm{m}$ microcell sized 9 $\mathrm{mm}^{2}$ sensors. The $36 \mathrm{~mm}^{2}$ sensor had the greatest sensor surface area and nearly $4 \mathrm{x}$ the number of microcells as the $35 \mu \mathrm{m}$ $9 \mathrm{~mm}^{2}$ sensor (Table S3) which likely contributed to its improved performance. The $50 \mu \mathrm{m}$ SPAD microcell size possessed an improved LOD compared to the SiPM with $35 \mu \mathrm{m}$ microcells. The increased microcell fill factor for the $50 \mu \mathrm{m}$ (72\%) compared to $35 \mu \mathrm{m}$ microcell array (64\%) likely contributed to the improved LOD. In comparison to the PMT, the LOD of the $36 \mathrm{~mm}^{2} \mathrm{SiPM}\left(4.91 \times 10^{-21} \mathrm{~mol}\right)$ was only about twice as high as the $48.1 \mathrm{~mm}^{2}$ PMT $\left(2.63 \times 10^{-21} \mathrm{~mol}\right)$.

\section{Dynamic Range and Signal Saturation}

The dynamic range of each sensor was measured using a calibration curve (Figure 3). The bias voltage for each SiPM was chosen by selecting that which provided the best SNR which also corresponded with the maximum bias voltage of the chips. The gain for the PMT was chosen as the point where SNR began to plateau with increasing signal (Figure 2, 350 $\mathrm{mV})$. Past this gain setting only small improvement in LOD could be achieved at significant loss to dynamic range. Standards were prepared spanning a concentration range of $10^{-12}$ to $10^{-5} \mathrm{M}$. As expected, at the highest fluorescein concentrations

(or emission intensities), the output from the SiPM detectors reaches a maximum number of cells that are activated (Figure S4). The high gain PMT setting and $9 \mathrm{~mm}^{2} 50 \mu \mathrm{m}$ and 36 $\mathrm{mm}^{2} 35 \mu \mathrm{m}$ SiPMs reached signal saturation at micromolar fluorescein concentrations. The lower capacitance $9 \mathrm{~mm}^{2}$ (35 $\mu \mathrm{m}$ microcell) SiPM did not show significant peak distortion due to saturation. The shorter deadtime of the smaller $9 \mathrm{~mm}^{2}$ sensor likely contributed to its improved dynamic range compared to the larger $36 \mathrm{~mm}^{2} \mathrm{SiPM}$. However, the larger microcells and larger sensor areas provided greater sensitivity.

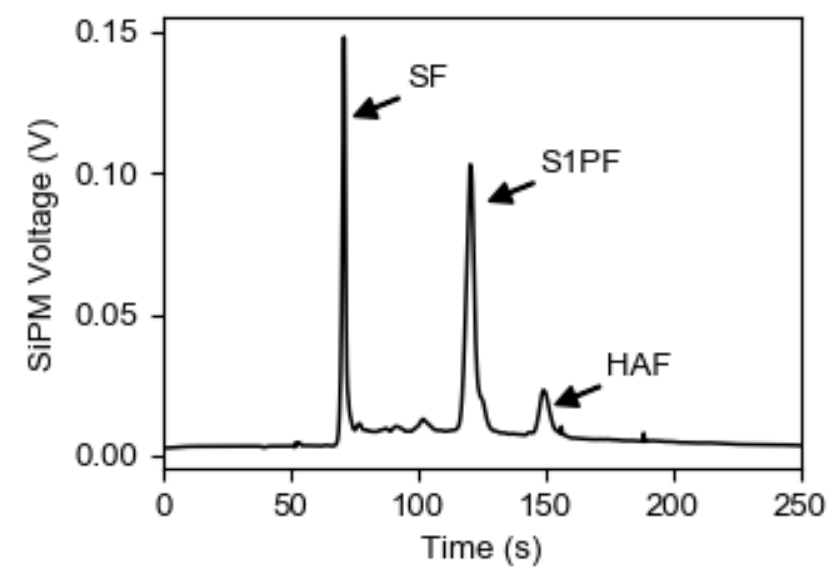

Figure 4. Electropherogram trace from a single cell loaded with SF. This assay measures the sphingosine kinase activity by quantifying the amount of reporter SF converted to S1PF by sphingosine kinase. Conversion of S1PF into HAF occurs due to the actions of sphingosine 1-phosphate lyase on S1PF.

\section{Single Cell Analysis}

Sphingosine kinase (SK) activity is often increased in a wide range of diseases, including Multiple Sclerosis, Alzheimer's, and various cancers and leukemias, among others. $^{22,23}$ SK inhibitors have also been shown to be lethal to cancer cells dependent on high SK activity. ${ }^{24}$ However, SK signaling can be highly heterogenous between cells of the same tumor sample. ${ }^{21}$ We have previously demonstrated that CE can be used to measure SK activity in single cells using the substrate SF. ${ }^{21}$ This fluorescent reporter consists of a sphingosine with a fluorescein conjugated to the acyl chain (SF) which is converted to S1PF by SK. ${ }^{25}$ Separation and quantification of the fluorescence of SF and S1PF from a single cell supplies a measure of the activity of SK in that cell. ${ }^{25}$ The $36 \mathrm{~mm}^{2}$ (35 $\mu \mathrm{m}$ microcell) with a $330 \Omega$ feedback resistance with a bias set to half its maximum $(-27.5 \mathrm{~V})$ was used to measure the SK activity in K562 leukemia (Figure 4). The detector was able to clearly detect the main sphingosine fluorescein and the sphingosine-1-phosphate fluorescein products necessary for the assay and demonstrates the ability of the SiPM to be applied to CE based chemical cytometry.

\section{CONCLUSION}

This work demonstrates that the SiPM can be used as a sensitive and affordable fluorescence detector for CE. The dynamic range required for chemical cytometry spans 3-5 orders of magnitude to match the range of the total amount of reporter loaded into a cell for peptide kinase reporters $\left(10^{-21}\right.$ to $10^{-19}$ mols) and for lipid kinase reporters $\left(10^{-19}\right.$ to $\left.10^{-17} \mathrm{mols}\right){ }^{3,21}$ The $\mathrm{SiPM}$ in this configuration has a dynamic range that spans five orders of magnitude while detecting as little as $10^{-12} \mathrm{M}$ or $10^{-21}$ moles of fluorescein. Thus, the SiPM detector possesses comparable performance characteristics as a PMT and is suitable for chemical cytometry assays by $\mathrm{CE}$. Commercially available SiPMs that are thermally regulated may provide an additional decrease in the noise compared to the detectors assessed here at a slightly increased system cost. An asset of the current SiPM chips is that the detectors can easily be configured according to the application need. This aspect provides flexibility for miniature, portable instrumentation, as well as micro- 
fluidic devices in situations where sensitivity, cost, and portability are important factors. ${ }^{21}$

\section{ACKNOWLEDGMENTS}

This work is supported by the National Institutes of Health grants R01CA233811, F31CA228223, and F31CA243312. The Authors would also recognize Pete Attayek for his contributions in designing the epi-illumination pathway.

\section{SUPPORTING INFORMATION}

Description of the custom CE instrumentation, list of materials for the fluorescence pathway and SiPM detector, SiPM detector circuit, schematic. Additional data describing the performance of the detector comparing SNR to detector bias, and traces of the detector's outputs at low and high intensity fluorescence.

\section{REFERENCES}

(1) Chen, D.; Dovichi, N. J. Single-Molecule Detection in Capillary Electrophoresis: Molecular Shot Noise as a Fundamental Limit to Chemical Analysis. Anal. Chem. 1996, 68 (4), 690-696. https://doi.org/10.1021/ac950651r.

(2) Cohen, D.; Dickerson, J. A.; Whitmore, C. D.; Turner, E. H.; Palcic, M. M.; Hindsgaul, O.; Dovichi, N. J. Chemical Cytometry: Fluorescence-Based Single-Cell Analysis. Annual Review of Analytical Chemistry 2008, 1 (1), 165-190. https://doi.org/10.1146/annurev.anchem.1.031207.113104.

(3) Vickerman, B. M.; Anttila, M. M.; Petersen, B. V.; Allbritton, N. L.; Lawrence, D. S. Design and Application of Sensors for Chemical Cytometry. ACS Chem. Biol. 2018, 13 (7), 1741-1751. https://doi.org/10.1021/acschembio.7b01009.

(4) Fang, X.-X.; Fang, P.; Pan, J.-Z.; Fang, Q. A Compact Short-Capillary Based High-Speed Capillary Electrophoresis Bioanalyzer. ELECTROPHORESIS 2016, 37 (17-18), 23762383. https://doi.org/10.1002/elps.201600195.

(5) Casto, L. D.; Do, K. B.; Baker, C. A. A Miniature 3D Printed LED-Induced Fluorescence Detector for Capillary Electrophoresis and Dual-Detector Taylor Dispersion Analysis. Anal. Chem. 2019. https://doi.org/10.1021/acs.analchem.8b05824.

(6) Kubáň, P.; Foret, F.; Erny, G. Open Source Capillary Electrophoresis. ELECTROPHORESIS 2019, 40 (1), 65-78. https://doi.org/10.1002/elps.201800304.

(7) Meacham, C. E.; Morrison, S. J. Tumour Heterogeneity and Cancer Cell Plasticity. Nature 2013, 501 (7467), 328-337. https://doi.org/10.1038/nature12624.

(8) Barker, N.; Bartfeld, S.; Clevers, H. Tissue-Resident Adult Stem Cell Populations of Rapidly Self-Renewing Organs. Cell Stem Cell 2010, 7 (6), 656-670. https://doi.org/10.1016/j.stem.2010.11.016.

(9) Yong Chen, D.; Dovichi, N. J. Yoctomole Detection Limit by Laser-Induced Fluorescence in Capillary Electrophoresis. Journal of Chromatography B: Biomedical Sciences and $\begin{array}{lllll}\text { Applications } & \mathbf{1 9 9 4}, & 657 & (2), & 265-269 .\end{array}$ https://doi.org/10.1016/0378-4347(94)00014-X.

(10) Galievsky, V. A.; Stasheuski, A. S.; Krylov, S. N. "Getting the Best Sensitivity from on-Capillary Fluorescence Detection in Capillary Electrophoresis" - A Tutorial. Analytica Chimica Acta 2016, 935, 58-81. https://doi.org/10.1016/j.aca.2016.06.015.

(11) Abraham, D. H.; Anttila, M. M.; Gallion, L. A.; Petersen, B. V.; Proctor, A.; Allbritton, N. L. Chapter Ten - Design of an Automated Capillary Electrophoresis Platform for SingleCell Analysis. In Methods in Enzymology; Allbritton, N. L., Kovarik, M. L., Eds.; Enzyme Activity in Single Cells; Aca-

demic Press, 2019; Vol. 628, pp 191-221. https://doi.org/10.1016/bs.mie.2019.06.016.

Mazzillo, M.; Condorelli, G.; Sanfilippo, D.; Valvo, G.; Carbone, B.; Fallica, G.; Billotta, S.; Belluso, M.; Bonanno, G.; Cosentino, L.; Pappalardo, A.; Finocchiaro, P. Silicon Photomultiplier Technology at STMicroelectronics. IEEE Transactions on Nuclear Science 2009, 56 (4), 2434-2442. https://doi.org/10.1109/TNS.2009.2024418.

Shin, Y.-H.; Barnett, J. Z.; Gutierrez-Wing, M. T.; Rusch, K. A.; Choi, J.-W. A Portable Fluorescent Sensing System Using Multiple LEDs. In Microfluidics, BioMEMS, and Medical Microsystems $X V$; International Society for Optics and Photonics, 2017; Vol. 10061, p 100610M. https://doi.org/10.1117/12.2261292.

(14) Santangelo, M. F.; Cono, S. L.; Vasquez, P.; Fallica, G.; Conoci, S.; Busacca, A. C.; Pagano, R.; Sciuto, E. L.; Lombardo, S.; Libertino, S. CY5 Fluorescence Measured with Silicon Photomultipliers. In 2014 IEEE Biomedical Circuits and Systems Conference (BioCAS) Proceedings; 2014; pp 284-287. https://doi.org/10.1109/BioCAS.2014.6981718.

Dorosz, P.; Baszczyk, M.; Kucewicz, W.; Mik, L. Silicon Photomultipliers Applied to the Fluorescence Detection of Biomarkers. Nuclear Instruments and Methods in Physics Research Section A: Accelerators, Spectrometers, Detectors and Associated Equipment 2018 https://doi.org/10.1016/j.nima.2018.10.156.

(16) C-Series SiPM Sensors: Silicon Photomultipliers (SiPM), Low-Noise, Blue-Sensitive. On Semiconductor.

(17) Modi, M.; Turner, G. C.; Podgorski, K. Two-Photon Imaging with Silicon Photomultipliers. bioRxiv 2019, 717850. https://doi.org/10.1101/717850.

(18) Santangelo, M. F.; Sciuto, E. L.; Busacca, A. C.; Petralia, S.; Conoci, S.; Libertino, S. SiPM as Miniaturised Optical Biosensor for DNA-Microarray Applications. Sensing and BioSensing Research 2015, 6, 95-98. https://doi.org/10.1016/j.sbsr.2015.08.003.

(19) Mik, Ł.; Stẹpień, J.; Jastrzạb, M.; Kucewicz, W.; Sapor, M. System for Low Intensity Fluorescence Light Measurement Based on Silicon Photomultiplier. In ICSES 2010 International Conference on Signals and Electronic Circuits; 2010; pp 383-386.

(20) Makkar, R. L.; Aliya, S. S.; Borse, V.; Srivastava, R. Design and Development of Portable Fluorescence Reader Using Silicon Photomultiplier (SiPM) Sensor. In Optical Sensing and Detection $V$; International Society for Optics and Photonics, 2018; Vol. 10680, p 106800B. https://doi.org/10.1117/12.2305846.

(21) Dickinson, A. J.; Hunsucker, S. A.; Armistead, P. M.; Allbritton, N. L. Single-Cell Sphingosine Kinase Activity Measurements in Primary Leukemia. Anal Bioanal Chem 2014, 406 (27), 7027-7036. https://doi.org/10.1007/s00216014-7974-6.

(22) Powell, J. A.; Wallington-Beddoe, C. T.; Pitson, S. M. Targeting Sphingosine Kinase 1 in Acute Myeloid Leukemia: Translation to Clinic. Int J Hematol Oncol 2017, 6 (2), 3134. https://doi.org/10.2217/ijh-2017-0011.

(23) Maceyka, M.; Harikumar, K. B.; Milstien, S.; Spiegel, S. Sphingosine-1-Phosphate Signaling and Its Role in Disease. Trends Cell Biol. 2012, 22 (1), 50-60. https://doi.org/10.1016/j.tcb.2011.09.003.

(24) Cao, M.; Ji, C.; Zhou, Y.; Huang, W.; Ni, W.; Tong, X.; Wei, J.-F. Sphingosine Kinase Inhibitors: A Patent Review. International Journal of Molecular Medicine 2018, 41 (5), 2450-2460. https://doi.org/10.3892/ijmm.2018.3505.

(25) Determination of Sphingosine Kinase Activity for Cellular Signaling Studies $\mid$ Analytical Chemistry https://pubs.acs.org/doi/full/10.1021/ac702305q (accessed Jul 16, 2020). 
